\title{
Initial Stability Of Semi-Submersible Vessel During Floating Onload Without Cargo
}

\author{
Quan Zhang \\ Navigation College \\ Dalian Maritime University \\ Dalian, China \\ dlzhangquan@163.com
}

\author{
Baijun Tian \\ Navigation College \\ Dalian Maritime University \\ Dalian, China \\ dltianbj@hotmail.com
}

\begin{abstract}
The initial stability of Semi-Submersible vessels, associated with transportation safety, can vary significantly during Floating Onload. There are some accidents happening in the past during operations of floating onload, and it highlights the requirement of analyzing the stability during this period in more detail. Based on ship statics, this paper took theoretical analysis of the semi-submersible vessel's hydrostatic data. It reveals that the number of GM can drop to the minimum, even to 0 or negative value, when the main deck submerged completely if the trim is 0 . However, when the semi-submersible vessel is floating onload in other floating conditions, the minimum GM can be improved and the rate of drop of GM can be decreased. Finally, this paper suggests that the semi-submersible vessel can improve the initial stability through altering its floating condition to trim by bow when the main deck begins to submerge.
\end{abstract}

KeyWords-Semi-Submersible Vessel; Initial Stability; Floating Onload; Ship Statics; Floating Condition

\section{INTRODUCTION}

With the rapid development of petroleum industry, more and more over-scaled equipments (such as offshore drilling platforms) have been constructed ${ }^{[1]}$. At present, only semi-submersible vessels are capable of transporting them efficiently and ensuring the transportation safety ${ }^{[2]}$.

Awkward cargoes can be loaded to semi-submersible vessels in a special way called floating onload. In the process of floating onload, there are three periods: before the main deck submerged, the main deck submerged completely and after the main deck submerged ${ }^{[3]}$. How to keep the initial stability of semi-submersible vessels in an appropriate range during floating onload is a problem.

At present, there are not many departments doing research on it. DNV (DET NORSKE VERITAS), CCS (China Classification Society) and Noble Denton set their own regulations and requirements respectively about calculating the stability of the semi-submersible vessel during floating onload and floating offload ${ }^{[4-6]}$. Hendrik D and Hannes, in their article "Stability Evaluation of SemiSubmersible Heavy Transport Vessels by a Progressive Flooding Simulation Tool", build up a semi-submersible vessel model by a software and simulate the process of discharging a drilling platform by means of floating offload " ${ }^{[7]}$. Frank V H, in his article "Semi-Submersible Heavy-Lift Ships in Operation", takes several successful transportation of ocean structures by semi-submersible vessels as examples to demonstrate how to guarantee the safety of ships and cargoes during transportation ${ }^{[8]}$. Wang Kuo, in his article "Research on Transportation and Offloading Performance of Spar Platform by SemiSubmersible Vessel", builds up a Spar platform model and semi-submersible vessel model by MOSES software to simulate the process of discharging the Spar platform by means of floating offload ${ }^{[9]}$. He calculates the strength and stability of the vessel under different arrangements of Spar platform and suggests a safe loading plan. Chen Wei, Zong Zhi, Lin Zhe, Hao Weiwei, in their article "A Preliminary Study on Intact Stability of Semi-Submersible Vessel", draw the GZ curve of a conversion semi-submersible during navigation ${ }^{[10]}$. All the articles above do not study how to guarantee appropriate initial stability during floating onload.

For guaranteeing the safety of floating onload, this paper is mainly going to study the initial stability based on an actual semi-submersible vessel during this period without cargoes due to the diversity of them. To reveal the trend of initial stability, it is going to suggest measures of improving the initial stability through theoretical analysis and calculation.

\section{PRINCIPAL VESSEl CHARACTERISTICS OF "H"}

Vessel " $\mathrm{H}$ " which is a newly built 50000DWT (Deadweight) self-propelled semi-submersible vessel is the example in this study. The principal characteristics of vessel " $\mathrm{H}$ " are listed in Table 1.

Table 1. The Principal Characteristics of Vessel "H"

\begin{tabular}{|l|l|}
\hline Length Overall & $221 \mathrm{~m}$ \\
\hline Moulded Breadth & $42 \mathrm{~m}$ \\
\hline Depth (To Main Deck) & $13.3 \mathrm{~m}$ \\
\hline Draught (Moulded Design) & $10 \mathrm{~m}$ \\
\hline Draught (Moulded Submerged) & $26 \mathrm{~m}$ \\
\hline Deadweight & $52789 \mathrm{t}$ \\
\hline
\end{tabular}

According to Table 1, Vessel "H" corresponds with all specifications of a standard modern self-propelled semi- 
submersible vessel. Therefore, Vessel " $\mathrm{H}$ " is suitable to be applied in this research.

\section{ANALYSIS OF INITIAL STABILITY DURING FLOATING ONLOAD ON EVEN KEEL}

The initial stability of the ship, measured by the initial metacentric height (GM), is determined by height of transverse metacenter (KM) and height of center of gravity $(\mathrm{KG})$. Initial metacentric height is defined as below ${ }^{[11]}$ :

$$
G M=K M-K B
$$

These three variables will be discussed respectively to figure out the trend of the initial stability during floating onload. Moreover, due to there are three periods during floating onload, the variables above will be analyzed in each period.

\section{A. Variation on $K M$}

Based on ship statics, $\mathrm{KM}$ is defined as below ${ }^{[11]}$ :

$$
K M=K B+\frac{I_{x}}{V}=K B+\frac{\alpha_{x} \cdot B^{2}}{d}
$$

With

KB_- height of center of buoyancy;

$\mathrm{I}_{\mathrm{x}}-$ moment of inertia;

$\mathrm{V}$ - volume;

$\alpha_{\mathrm{x}}-$ moment of inertia coefficient;

B - moulded breadth;

d-draught;

It can be seen in Figure 1, due to the block below the main deck of the semi-submersible vessel is a regular boxlike structure, the number of B only increases slightly over draught at bow and stern before the main deck submerged. However, $d$ increases linearly and its rate of increase is larger than $\mathrm{B}$. Therefore, $\mathrm{B}^{2} / \mathrm{d}$ decreases sharply over draught. Moreover, KB increases linearly, which is similar to $\mathrm{d}$. but later is much greater. The reason is that as center of buoyancy is the geometric center of volume, the number of $\mathrm{KB}$ is approximately equal to $0.5 \mathrm{~d}$ for regular block. In addition, the rate of increase of $\mathrm{KB}$ is about half of it of $\mathrm{d}$. Finally, the rate of decrease of $\mathrm{B}^{2} / \mathrm{d}$ is almost as twice as the rate of increase of $\mathrm{KB}$. As a result, it causes $\mathrm{KM}$ decrease gradually.
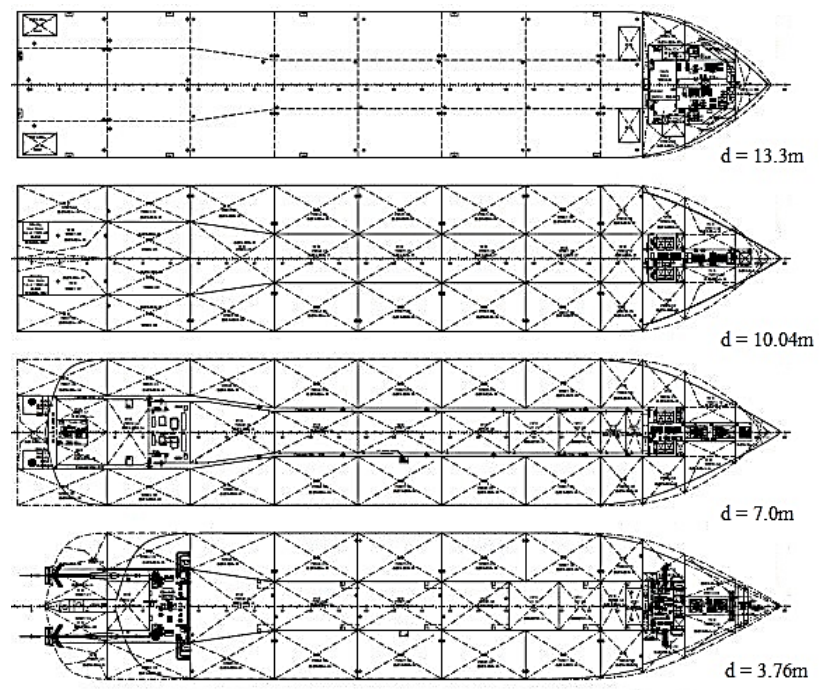

Figure 1. Waterplane of Vessel " $\mathrm{H}$ " before main deck submerged
At the moment of main deck submerged, due to the draught changes little, $\mathrm{V}$ and $\mathrm{KB}$ almost remain the same in comparison with them before the main deck submerged (See Table 2). However, it can be seen in Figure 2 that as complete main deck has been submerged, the area of waterplane can only be supplied by bow structure and columns astern. As a result, $\mathrm{I}_{\mathrm{x}}$, which is in proportion with area of waterplane, drops dramatically when main deck submerged, and makes KM decrease rapidly ${ }^{[9]}$.

Table 2. Numbers of variables affecting KM when main deck submerged

\begin{tabular}{|c|c|c|c|c|}
\hline $\mathrm{d}$ & KB & $\mathrm{I}_{\mathrm{x}}$ & $\mathrm{V}$ & $\mathrm{KM}$ \\
\hline $13.3 \mathrm{~m}$ & $7.02 \mathrm{~m}$ & $1175055.63 \mathrm{~m}^{4}$ & $101385.3 \mathrm{~m}^{3}$ & $18.61 \mathrm{~m}$ \\
\hline $13.4 \mathrm{~m}$ & $7.03 \mathrm{~m}$ & $117727.7 \mathrm{~m}^{4}$ & $101489.4 \mathrm{~m}^{3}$ & $8.19 \mathrm{~m}$ \\
\hline
\end{tabular}
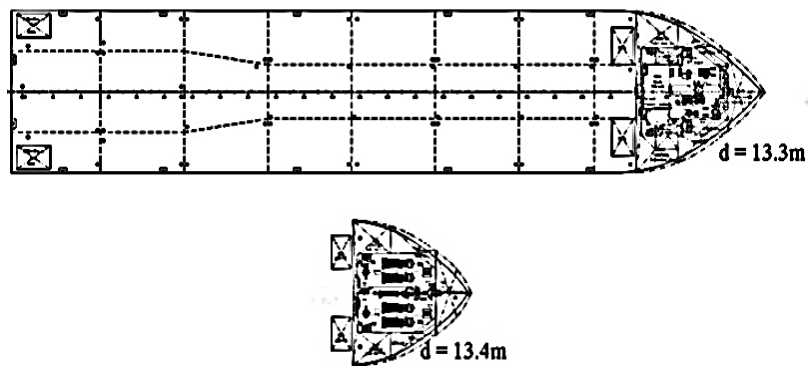

Figure 2. Waterplane of Vessel "H" when main deck submerged

After main deck submerged, $\mathrm{KB}$ and $\mathrm{d}$ continue increasing. Moreover, as shown in Figure 3, for compensating $I_{x}$ which drops rapidly due to main deck submerged, the ship design institute usually makes area of waterplane of the forecastle increase over draught by designing forecastle as an inverse trapezium-like structure. It could cause $\mathrm{B}$ increase significantly. Therefore, $\mathrm{B}^{2} / \mathrm{d}$ increases gradually over draught. As both $\mathrm{B}^{2} / \mathrm{d}$ and $\mathrm{KB}$ increase over draught, KM rebounds in a small degree.
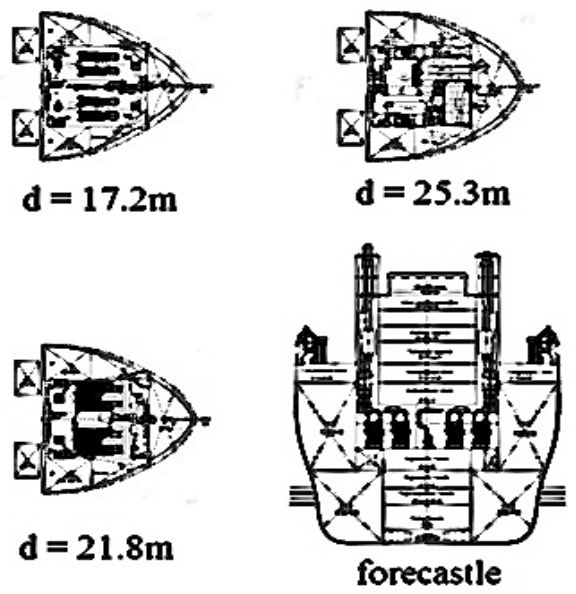

Figure 3. Waterplane of Vessel " $H$ " after main deck submerged and the inverse trapezium-like forecastle

Due to the discussion above, it can be concluded that the variation on KM contains three phases. Firstly, the number of KM declines gradually over draught before the main deck submerged. Secondly, when the draught reaches to the height of the main deck, the number of KM drops significantly to the minimum because the main deck submerged completely. Finally, after submerging of the 
main deck, the number of KM increases slightly over draught. Figure 4 showed the KM curve of Vessel " $H$ " on even keel.

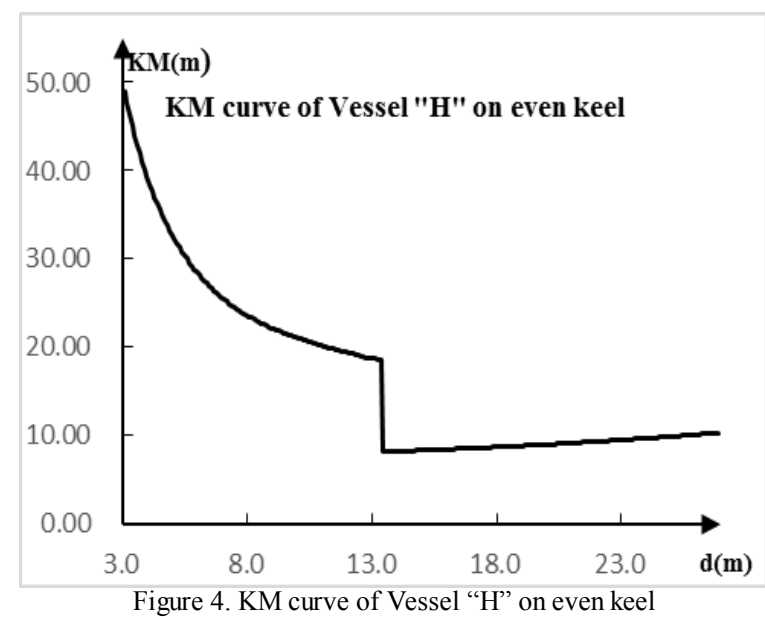

\section{B. Variation on $K G$}

Based on ship statics, $\mathrm{KG}$, the other variable affecting $\mathrm{GM}$, is defined as below ${ }^{[11]}$ :

$$
K G=\frac{W_{0} K G_{0}+\sum_{i=1}^{n} P_{i} K G_{i}}{W}
$$

With

$\mathrm{W}_{0}$ - light weight of vessel;

$\mathrm{KG}_{0}$ height of center of gravity (light weight of vessel);

$\mathrm{n}$-number of items composing the gross weight of vessel;

$\mathrm{P}_{\mathrm{i}}$-weight of number i item; item);

$\mathrm{KG}_{\overline{1}}$-height of center of gravity (weight of number $\mathrm{i}$

$\mathrm{W}$ — gross weight of vessel;

The variation on KG is not as complex as KM. Due to the height of forecastle of the semi-submersible vessel is usually big, the number of $\mathrm{KG}_{0}$ is large. During the period of floating onload, the ballasting sequence is generally from lower ballast tanks to upper. It causes that at beginning, number of $\mathrm{KG}_{\mathrm{i}}$ is less than it of $\mathrm{KG}_{0}$, and then number of $\mathrm{KG}_{\mathrm{i}}$ is greater than it of $\mathrm{KG}_{0}$. As a result, number of $\mathrm{KG}$ declines firstly and then rises gradually due to the Equation 3. The $\mathrm{KG}$ curve, which is based on a ballasting process of Vessel " $\mathrm{H}$ ", can be drawn by loading computer and be shown in Figure 5.

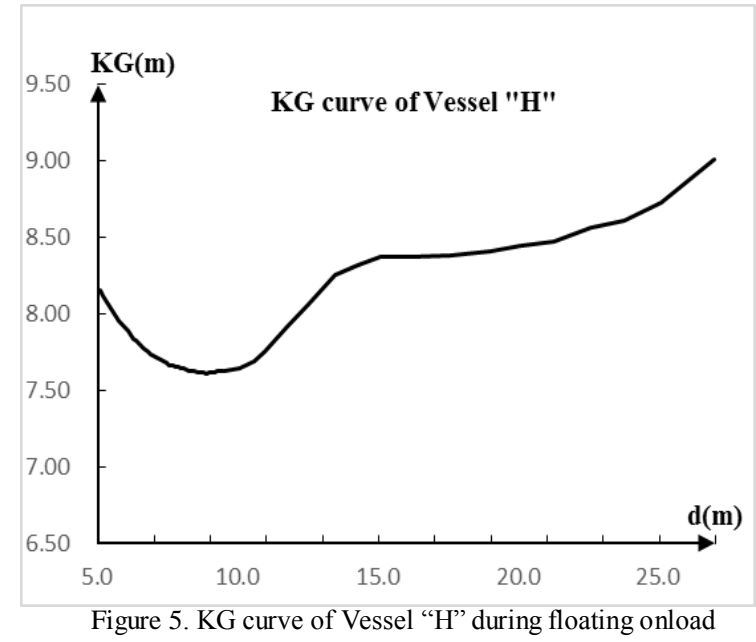

\section{Variation on $G M$}

According to the Figure 4 and Figure 5, it can be seen that the variation on $\mathrm{KM}$ was much sharper than $\mathrm{KG}$, and the number of $\mathrm{KM}$ was also larger than KG. Therefore, this paper infers that GM is mainly affected by KM during floating onload and the trend of GM curve should be identical to KM. The GM curve of Vessel " $H$ " is shown in Figure 6.

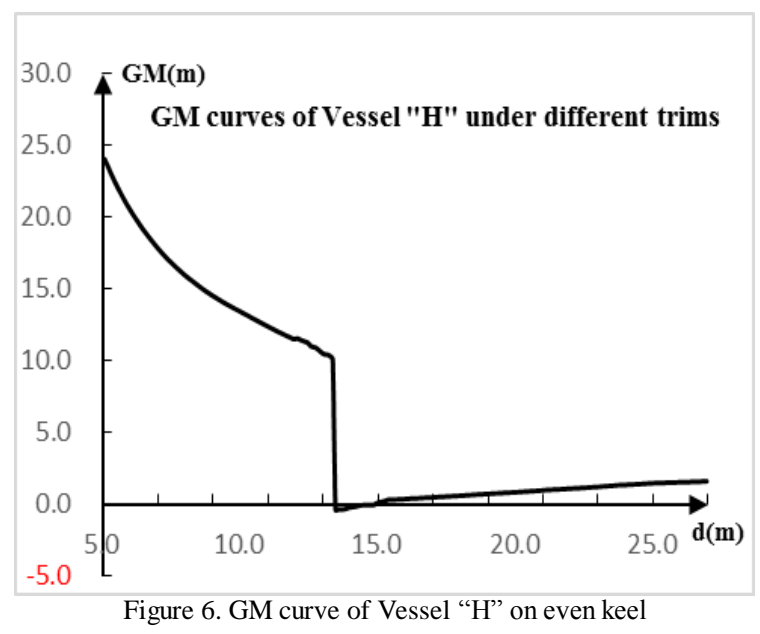

As shown in the Figure 6, the trend of GM curve was exactly same to it of KM. In addition, the Figure 6 pointed out that at the moment of the main deck submerged completely, the number of GM dropped to the minimum and even less than 0 . On the basis of ship statics, negative GM can lead to the vessel capsize even if there is only small disturbance on it. Therefore, some measures must be taken to avoid this phenomenon, and to keep GM in an appropriate positive range.

\section{MEASURES TO IMPROVE THE INITIAL STABILITY DURING FLOATING ONLOAD}

Due to the semi-submersible vessel is ballasted to floating onload on even keel, the total main deck is suddenly submerged when the draught arrives at the height of the main deck. It can cause that the main deck no longer composite the area of waterplane. However, if the semisubmersible vessel can alter its floating condition when the 
main deck begins to submerge, the status of drop of GM will be improved obviously.

When the semi-submersible vessel proceeds floating onload by trim, the rate of drop of KM decreases obviously in the period of main deck submerging. The reason for this phenomenon is that the rate of main deck submerging by trim is smaller than it under even keel, and the greater number of trim, the smaller rate. It can directly prevent the rate of decline of waterplane from decreasing sharply, and lead to the rate of drop of $I_{x}$ slows down. Therefore, the rate of drop of KM becomes smaller by trim. Furthermore, with the absolute number of trim rised, the midship draught increased at the moment of main deck submerged completely. It could cause that number of B becomes greater because of the inverse trapezium-like forecastle. According to the Equation 2, the greater number of $\mathrm{B}$ makes the greater number of $\mathrm{KM}$. As a result, the minimum number of $\mathrm{KM}$ increases slightly because the absolute number of trim is rising. And it can lead to the minimum number of GM increase.

In addition, although the absolute numbers of trim are equal, the minimum number of GM under trim by bow is different from it under trim by stern. The reason is that the center of floatation moves forward from midship to bow due to the larger area of waterplane of bow. Based on ship statics, the center of floatation is on axis of trim under the condition of trim by equal volume. It can cause that the submerging of main deck under trim by stern occurs earlier than trim by bow. Therefore, when main deck submerged completely, the midship draught under trim by stern is slightly less than it under trim by bow. Because of the inverse trapezium-like forecastle, the number of B under trim by bow is larger. It could lead that the minimum KM under trim by bow is larger than it under trim by stern. Consequently, the minimum GM under trim by bow is greater.

However, either before or after main deck submerged, the variation on KM under trim 0 was exactly same to it in other floating conditions. Due to the block below main deck of semi-submersible vessel is a regular box-like structure and the forecastle is a regular inverse trapeziumlike structure, when the midship draught is fixed, the area of waterplane and the volume are almost identical. It causes that, as Table 3 and Table 4 pointed out, numbers of $\mathrm{V}$, B and $\mathrm{KB}$ are nearly equal under different trims, respectively. As a result, the numbers of KM are nearly the same, and the numbers of GM in different floating conditions are approximately equal too.

Table 3. Numbers of variables affecting KM before main deck submerged completely under different trims

\begin{tabular}{|c|c|c|c|c|}
\multicolumn{5}{|c|}{$(\mathrm{d}=8.0 \mathrm{~m})$} \\
\hline trim & $-2.0 \mathrm{~m}$ & 0 & $2.0 \mathrm{~m}$ & $4.0 \mathrm{~m}$ \\
\hline $\mathrm{I}_{\mathrm{x}}$ & $1.12^{*} 10^{6} \mathrm{~m}^{4}$ & $1.10^{*} 10^{6} \mathrm{~m}^{4}$ & $1.08 * 10^{6} \mathrm{~m}^{4}$ & $1.06^{*} 10^{6} \mathrm{~m}^{4}$ \\
\hline $\mathrm{V}$ & $57643.4 \mathrm{~m}^{3}$ & $57121.9 \mathrm{~m}^{3}$ & $56763.7 \mathrm{~m}^{3}$ & $56562.2 \mathrm{~m}^{3}$ \\
\hline $\mathrm{KB}$ & $4.25 \mathrm{~m}$ & $4.20 \mathrm{~m}$ & $4.19 \mathrm{~m}$ & $4.23 \mathrm{~m}$ \\
\hline
\end{tabular}

Table 4. Numbers of variables affecting KM after main deck submerged completely under different trims

\begin{tabular}{|c|c|c|c|c|}
\multicolumn{5}{c|}{$(\mathrm{d}=16.0 \mathrm{~m})$} \\
\hline trim & $-2.0 \mathrm{~m}$ & 0 & $2.0 \mathrm{~m}$ & $4.0 \mathrm{~m}$ \\
\hline $\mathrm{I}_{\mathrm{x}}$ & $1.25^{*} 10^{5} \mathrm{~m}^{4}$ & $1.28 * 10^{5} \mathrm{~m}^{4}$ & $1.31 * 10^{5} \mathrm{~m}^{4}$ & $1.35^{*} 10^{5} \mathrm{~m}^{4}$ \\
\hline $\mathrm{V}$ & $1.04 * 10^{5} \mathrm{~m}^{3}$ & $1.04 * 10^{5} \mathrm{~m}^{3}$ & $1.05^{*} 10^{5} \mathrm{~m}^{3}$ & $1.06^{*} 10^{5} \mathrm{~m}^{3}$ \\
\hline $\mathrm{KB}$ & $7.18 \mathrm{~m}$ & $7.24 \mathrm{~m}$ & $7.30 \mathrm{~m}$ & $7.36 \mathrm{~m}$ \\
\hline
\end{tabular}

Figure 7 and Figure 8 showed the KM curves and GM curves respectively under different trims. It can be seen that both numbers of KM and GM were almost same under the identical midship draught before and after the main deck submerged. However, the slope of these curves in the period of the main deck submerged completely were different. Furthermore, the minimum number of GM became positive due to floating onload by trim. When the absolute numbers of trim were equal, both the minimum GM and minimum KM under trim by bow were slightly bigger than them respectively under trim by stern.

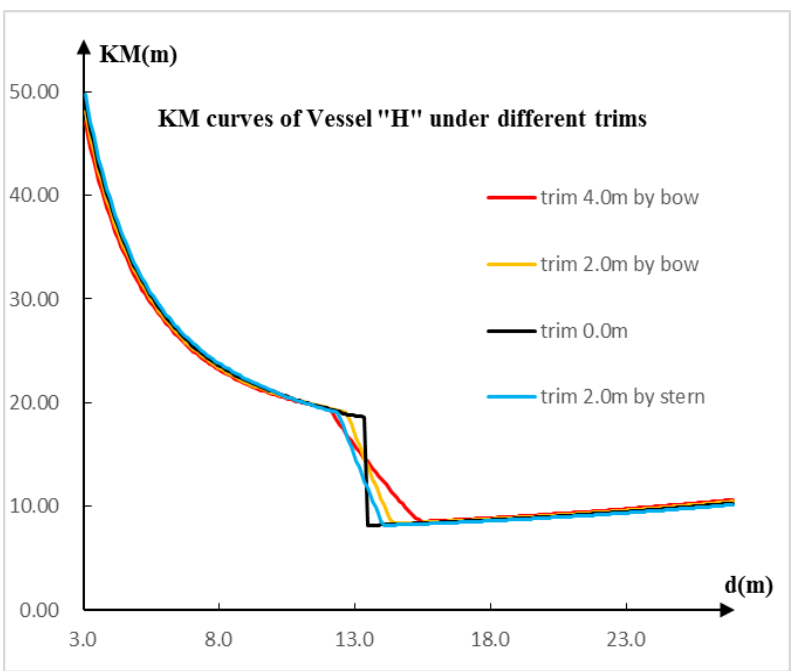

Figure 7. KM curves of Vessel "H" under different trims

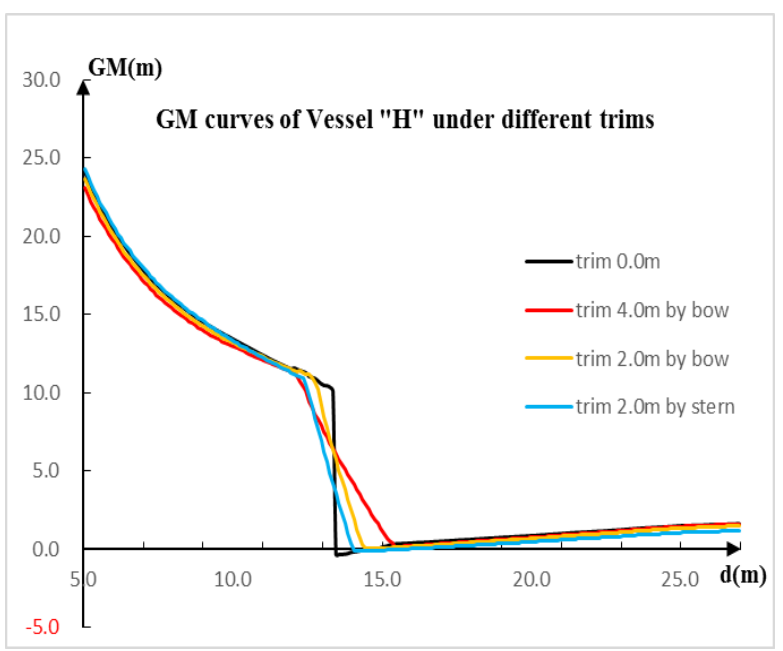

Figure 8. GM curves of Vessel " $\mathrm{H}$ " under different trims 


\section{CONCLUSION}

As discussed before, whatever trim is, it concludes that the number of GM would decrease gradually over draught before main deck submerged, and whatever the trim is, numbers of GM under the identical midship draught are nearly same. In the period of main deck submerging, the number of GM drops dramatically to the minimum. As the greater absolute number of trim can prevent the rate of drop of $I_{x}$ from dropping sharply and then the number of $B$ increase, the rate of drop of GM will slow down and the minimum GM will become greater. However, if the absolute numbers of trim are equal, the minimum GM under trim by bow is slightly greater than it under trim by stern with the reason of the center of floatation moving forward from midship to bow. After main deck submerged, the number of GM picks up over draught because of the inverse trapezium-like forecastle.

In the process of floating onload, it can be suggested to submerge on even keel firstly and do not alter floating condition to trim by bow until main deck begins to submerge. After main deck submerged completely, the floating condition can be recovered to even keel. Finally, floating condition should be kept till the designated draught. The method above can improve the minimum GM and slow down the rate of drop of GM during main deck submerged. Consequently, it can guarantee the safety of floating onload.

\section{REFERENCES}

[1] Jiao Yuqing. Summarization of General Design of 50000 DWTSemi-submersible Heavy Lift Vessel [J]. Journal of Ship Design, 2010, (123): 58-64.

[2] Su Chen. The Study on the Safety Control of Semi-submersible Ship Carrying Huge Heavy Cargo [D]. LiaoNing: Dalian Maritime University, 2012.

[3] Chen Baiqiao. Feasibility Analysis of Ocean Structures Transportation by Semi-submersibles and Ballasting Optimization [D]. TianJin: Tianjin University, 2009.

[4] DNV. Rules for classification of ships. 2012

[5] GL Noble Denton. Guidelines for marine transportation. 2010.

[6] CCS. Rules for classification of sea-going steel ships. 2014.

[7] Hendrik D,Hannes H.Stability. Evaluation of Semi-Submersible Heavy Transport Vessels by a progressive flooding simulation tool. OMAE, 2012:1-9.

[8] Frank V H. Semi-Submersible Heavy-Lift Ships in operation. SenW 57STE JAARGANG NR 8, 1991:448-453.

[9] Wang Kuo. Research on Transportation and Offloading Performance of Spar Platform by Semi-Submersible Vessel [D]. TianJin: Tianjin University, 2010.

[10] Chen Wei, Zong Zhi, Lin Zhe, Hao Weiwei. A Preliminary Study on Intact Stability of Semi - submersible Vessel [J]. Chinese Journal of Ship Research, 2009,4(3):13-23.

[11] Du Jiali, Jiang Hua. Ship Principles [M]. $1^{\text {st }}$ ed. Dalian: Dalian Maritime University Press, 2011. 